\title{
REVIEW \\ Prediction and estimation of effective population size
}

\author{
$\mathrm{J} \mathrm{Wang}^{1}$, E Santiago ${ }^{2}$ and A Caballero ${ }^{3}$
}

Effective population size $\left(N_{e}\right)$ is a key parameter in population genetics. It has important applications in evolutionary biology, conservation genetics and plant and animal breeding, because it measures the rates of genetic drift and inbreeding and affects the efficacy of systematic evolutionary forces, such as mutation, selection and migration. We review the developments in predictive equations and estimation methodologies of effective size. In the prediction part, we focus on the equations for populations with different modes of reproduction, for populations under selection for unlinked or linked loci and for the specific applications to conservation genetics. In the estimation part, we focus on methods developed for estimating the current or recent effective size from molecular marker or sequence data. We discuss some underdeveloped areas in predicting and estimating $N_{e}$ for future research.

Heredity (2016) 117, 193-206; doi:10.1038/hdy.2016.43; published online 29 June 2016

\section{INTRODUCTION}

The concept of effective population size, introduced by Sewall Wright (1931, 1933), is central to plant and animal breeding (Falconer and Mackay, 1996), conservation genetics (Frankham et al., 2010; Allendorf et al., 2013) and molecular variation and evolution (Charlesworth and Charlesworth, 2010), as it quantifies the magnitude of genetic drift and inbreeding in real-world populations. A substantial number of extensions to the basic theory and predictions were made since the seminal work of Wright, with main early developments by James Crow and Motoo Kimura (Kimura and Crow, 1963a; Crow and Kimura, 1970) and later by a list of contributors. Several review papers (Crow and Denniston, 1988; Caballero, 1994; Wang and Caballero, 1999; Nomura, 2005a) and population genetic books (Fisher, 1965; Wright, 1969; Ewens, 1979; Nagylaki, 1992) have summarised the existing theory in predicting the effective size of a population at different spatial and timescales under various inheritance modes and demographies. Comparatively, methodological developments (reviewed by Schwartz et al., 1999; Beaumont, 2003a; Wang, 2005; Palstra and Ruzzante, 2008; Luikart et al., 2010; Gilbert and Whitlock, 2015) in estimating the effective size of natural populations from genetic data lag behind but are accelerating in the past decade, thanks to the rapid developments of molecular biology.

The classical developments of effective population size theory are based on the rate of change in gene frequency variance (genetic drift) or the rate of inbreeding. The effective population size is defined in reference to the Wright-Fisher idealised population, that is, a hypothetical population with very simplifying characteristics where genetic drift is the only factor in operation, and the dynamics of allelic and genotypic frequencies across generations merely depend on the population census $(N)$ size. The effective size of a real population is then defined as the size of an idealised population, which would give rise to the rate of inbreeding and the rate of change in variance of gene frequencies actually observed in the population under consideration, which correspond to the so-called inbreeding and variance effective sizes, respectively (Crow and Kimura, 1970).

Predictions of the effective population size can also be obtained from the largest nonunit eigenvalue of the transition matrix of a Markov Chain which describes the dynamics of allele frequencies. Such derived effective size is called as eigenvalue effective size (see Ewens, 1979, pp. 104-112), which is equivalent to the random extinction effective size (Crow, 1954; see also Haldane, 1939). The transition matrix can be written for many genetic models and is particularly useful for complex scenarios such as populations varying in size, having age structures or being subject to demographic changes (for example, Charlesworth, 2001; Pollak, 2002; Wang and Pollak, 2002; Engen et al., 2005). A less often used approach is that for the mutation effective size, defined by the probability of identity in state of genes rather than identity by descent under an infinite allele model of mutations with a defined mutation rate (Whitlock and Barton, 1997).

Later developments based on coalescence theory (Wakeley, 2008) have also proved to be useful in the prediction of effective population size, particularly in the evolutionary context for predicting genetic variability at the molecular level (Charlesworth, 2009; Nicolaisen and Desai, 2012, 2013). The coalescence theory states that the chance of coalescence of any two random gene copies in one generation time is $1 / 2 N$, which is the same as the rate of increase in identity by descent occurred from one generation to the next one. Thus, the probability of coalescence $t$ generations ago is $(1-(1 / 2 N))^{t-1}(1 / 2 N)$. Therefore, the average time of coalescence of two randomly chosen genes is $T=2 \mathrm{~N}$. The coalescent effective population size refers to the expected time of coalescence $T$, in generations, of gene copies such that $T=2 N_{e}$ (Nordborg and Krone, 2002; Wakeley and Sargsyan, 2009).

In this paper, we present a general overview of the main developments for predicting the effective population size $\left(N_{e}\right)$. The review does not attempt to be exhaustive, and some of the material mentioned in previous reviews will not be repeated. We mainly focus

\footnotetext{
${ }^{1}$ Institute of Zoology, Zoological Society of London, London, UK; ${ }^{2}$ Departamento de Biología Funcional, Facultad de Biología, Universidad de Oviedo, Oviedo, Spain and ${ }^{3}$ Departamento de Bioquímica, Genética e Inmunología, Facultad de Biología, Universidad de Vigo, Vigo, Spain Correspondence: Dr J Wang, Institute of Zoology, Zoological Society of London, Regent's Park, London NW1 4RY, UK. E-mail: jinliang.wang@ioz.ac.uk Received 29 September 2015; revised 3 May 2016; accepted 16 May 2016; published online 29 June 2016
} 
on populations with different modes of reproduction, populations under selection and populations under genetic management in captive breeding conservation programmes, complementing previous reviews and adding material not covered or only partially covered by them. We also review the developments in estimating contemporary effective sizes from genetic marker data, focussing on the estimation principles and ignoring the technical details that were covered in the original papers. The underlying assumptions, application scopes, robustness and accuracies of different estimation methods are discussed and compared.

\section{PREDICTION OF THE EFFECTIVE POPULATION SIZE}

In this section, we will summarise the main predictive equations for the asymptotic effective population size reached after a number of generations in a regular breeding system. In this case, all of the above approaches generally lead to the same predictive equations of $N_{e}$, except for a few particular scenarios. For example, in a regular breeding system for an undivided population the asymptotic inbreeding and variance effective sizes converge. Only in situations such as when the population is subdivided permanently in independent sublines with completely independent pedigrees (Wang, 1997a, b) or when the population is decreasing or increasing in size, these types of $N_{e}$ will differ permanently. In fact, many of the equations shown below have been derived by two or more of the above approaches, and we will only mention some of these. For clarity and a better understanding of the main principles, several simplifying assumptions will also be made in this prediction section. Unless otherwise stated, we will assume that populations do not change size through time and are large enough so that second-order terms of $1 / N_{e}$ can be safely ignored. These terms are generally of little relevance but make the derivations and the $N_{e}$ equations rather cumbersome. Finally, a single undivided population with discrete generations under a regular breeding scheme will be generally assumed unless otherwise indicated, so that prediction equations refer to asymptotic (Caballero, 1994) effective population sizes.

\section{Populations with different modes of reproduction}

As a starting point, we consider the simple equation derived by Wright (1938), which takes account of the variance of the contributions from parents to progeny $\left(S_{k}^{2}\right)$ in a population of constant size $N$,

$$
N_{e}=\frac{4 N}{2+S_{k}^{2}} \text {. }
$$

This expression also assumes a population either containing only hermaphrodites or comprising equal numbers of males and females, diploid autosomal inheritance and random mating (including selfing for hermaphrodites). In Equation (1), the term $S_{k}^{2}$ accounts for the genetic drift caused by the variable contributions among parents, whereas the first term ' 2 ' in the denominator accounts for the genetic drift caused by the Mendelian segregation of heterozygotes (that is, the drift in allele frequency arising from the fact that the progeny from a heterozygote can alternatively receive one or the other allele). It can also be seen as the variance in contributions between paternal and maternal genes at a locus within an individual or part of the variance in contribution between grandparents (the term $\delta^{2}$ in Equation (2) of Wang and Hill (2000)).

An illustrative generalisation of Equation (1) to the case of different numbers of males $\left(N_{m}\right)$ and females $\left(N_{f}\right)$ is

$$
N_{e}=\frac{16 N_{m} N_{f} /\left(N_{m}+N_{f}\right)}{2+S_{k}^{2}},
$$

with

$$
\begin{aligned}
S_{k}^{2}= & \frac{N_{f}}{N_{m}+N_{f}}\left[S_{m m}^{2}+2\left(\frac{N_{m}}{N_{f}}\right) S_{m m, m f}+\left(\frac{N_{m}}{N_{f}}\right)^{2} S_{m f}^{2}\right] \\
& +\frac{N_{m}}{N_{m}+N_{f}}\left[S_{f f}^{2}+2\left(\frac{N_{f}}{N_{m}}\right) S_{f m, f f}+\left(\frac{N_{f}}{N_{m}}\right)^{2} S_{f m}^{2}\right],
\end{aligned}
$$

where $S_{x y}^{2}$ is the variance of the number of offspring of sex $y$ from parents of sex $x$ and $S_{x m, x f}$ is the covariance of the numbers of male and female offspring from parents of sex $x$. Equation (2) is the same as that derived by Hill (1979), although it is expressed in a different form. It reduces to the classical equation of Wright $(1933,1939)$ for a Poisson distribution of progeny number (that is, $S_{x y}^{2}=N_{y} / N_{x}, S_{x m, x f}=0$ for $\operatorname{sex} x, y=m, f)$,

$$
N_{e}=\frac{4 N_{m} N_{f}}{N_{m}+N_{f}},
$$

which shows that unequal numbers of males and females in a population introduce a systematic variance in contribution between male and female parents and thus a reduction in effective size.

Predictive formulae for the effective size of X-linked genes were originally given by Wright (1933) and later extended by other authors (see Caballero, 1995). Later developments have also been made for Y-linked and maternally transmitted genes (Charlesworth, 2001; Laporte and Charlesworth, 2002; Evans and Charlesworth, 2013).

The generalisation of Equation (1) to the case of a partially selfed population (in which there is partial selfing with proportion $\beta$, random mating otherwise) is

$$
N_{e}=\frac{4 N}{2(1-\alpha)+S_{k}^{2}(1+\alpha)},
$$

(Crow and Morton, 1955), where

$$
\alpha=\beta /(2-\beta)
$$

(Haldane, 1924) quantifies the deviation from Hardy-Weinberg equilibrium or the correlation of genes within individuals relative to the genes taken at random from the population (Wright, 1969). The value of $\alpha$ in a large random mating population is approximately 0 (slightly negative when second-order terms are considered; see Equation (23) below and Wang, 1996a). For the case of biparental inbreeding such as partial full-sib mating in dioecious species, the expression is the same as Equation (5) except that the term $(1+\alpha)$ should be replaced by $(1+3 \alpha)$ (Caballero and Hill, 1992). The equilibrium value of $\alpha$ for biparental inbreeding is also different from Equation (6) (for example, Ghai, 1969).

The generalisation of Equation (5) for different numbers of males and females was made by Wang (1996b), adding some covariance terms not considered by Caballero (1994) (Equation (17)). Equation by Wang (1996b) also allows for different numbers of males and females varying over generations.

An excess of inbred matings ( $\alpha>0$ in Equation (5)) has the effect of increasing the correlation of genes within individuals and decreasing the frequency of heterozygotes by a factor of $\alpha$. It results in a decrease in the genetic drift owing to a decrease in Mendelian segregation variance and an increase in the genetic drift owing to an increase in the variance of contributions among individuals. Compared with random mating $(\alpha=0)$, whether an excess $(\alpha>0)$ or a deficit $(\alpha<0)$ of inbred matings may increase or decrease $N_{e}$ depends on the variance in family size, $S_{k}^{2}$. For the case of partial selfing (Equation (5)), inbreeding $(\alpha>0)$ increases $N_{e}$ when $S_{k}^{2}<2$ and decreases $N_{e}$ when $S_{k}^{2}>2$. At exactly $S_{k}^{2}=2$, selfing has no effect on $N_{e}$. 
Predictions of the effective size for X-linked genes in non-random mating populations were given by Wang (1996c). Nomura (2002a, 2005b) also provided predictions of the effective size for a variety of mating systems in animals (see also Balloux and Lehmann (2003)). For example, for harem polygamy, where successful males generally mate with most or all of the females in their harem and the females generally mate with only one male, the effective size, for a Poisson distribution of progeny number, is better approximated by

$$
N_{e}=\frac{4 N_{m} N_{f}}{2 N_{m}+N_{f}}
$$

rather than by Equation (4), showing the larger impact of male number for this type of mating system. Other predictions of $N_{e}$ for different mating systems and overlapping generations have been provided by Nunney (1993).

Equation (5) can also be obtained following the concept of longterm contributions from ancestors to descendants developed by Wray and Thompson (1990) in the context of populations under selection. As suggested by Woolliams and Thompson (1994) and shown by Caballero and Toro $(2000,2002)$, the expressions can be approximated by

$$
N_{e} \approx \frac{2 N}{\left(1+V_{\infty}\right)(1-\alpha)},
$$

where $V_{\infty}$ is the variance of long-term contributions from ancestors to descendants. For random mating $(\alpha=0), V_{\infty}=1$ and $N_{e}=N$, as expected.

For a proportion $\beta$ of partial selfing, $V_{\infty} \approx S_{k}^{2} /[2(1-\beta)]$ if $\alpha$ is not too close to 1 (Caballero and Toro, 2000), which, when substituted into Equation (8), gives Equation (5). When the numbers of selfed and nonselfed progeny are independently Poisson distributed, $S_{k}^{2} \approx 2+2 \beta$ (see Nomura, 1999a for a more precise prediction), and both Equations (5) and (8) reduce to

$$
N_{e}=\frac{N}{1+\alpha}
$$

(Li, 1976, p. 562; Pollak, 1987; Caballero and Hill, 1992; Nordborg and Krone, 2002). For a population with Poisson distribution of family size and complete selfing, Equation (9) reduces to $N_{e}=N / 2$. Equation (8) can also be applied to the scenario of partial full-sib mating with the appropriate approximation for $V_{\infty}$ (Caballero and Toro, 2000).

Predictions of the effective size for populations with mixed sexual and asexual reproduction systems and discrete and overlapping generations have been developed by Yonezawa (1997). Assuming a monoecious plant species carrying out asexual propagation with a proportion $\delta$ in a population of constant size $N$ (that is, an average progeny number of one and two for asexual propagation and sexual reproduction, respectively), Equation (5) of Yonezawa (1997) can be rearranged to

$$
N_{e}=\frac{4 N}{\left[2(1-\alpha)+S_{k}^{2}(1+\alpha)\right](1-\delta)+4 \delta S_{c}^{2}(1+\alpha)},
$$

where $S_{c}^{2}$ is the variance of the number of asexually produced progeny among plants. If there is no asexual reproduction $(\delta=0)$, Equation (10) reduces to Equation (5), as it should. If all reproduction is asexual $(\delta=1)$, Equation (10) reduces to

$$
N_{e}=\frac{N}{S_{c}^{2}(1+\alpha)} \text {. }
$$

Interestingly, if the number of asexually produced progeny is Poisson distributed $\left(S_{c}^{2}=1\right)$, the expression is the same as for a sexually reproducing partially selfed population where the numbers of selfed and nonselfed progeny are independently Poisson distributed, that is, Equation (9). If all individuals are homozygotes $(\alpha=1), N_{e}=N / 2$, the same as for a fully selfed population.

An extension of Equation (10) to overlapping generations was also given by Yonezawa (1997). Equation (10) assumes that sexual and asexual contributions are independent. Predictions relaxing this assumption and extensions to more complex models were given by Yonezawa et al. $(2000,2004)$. Analytical expressions for these models of mixed sexual and asexual species were also given by Orive (1993) and Balloux et al. (2003) using coalescence theory.

Predictions of effective size for haplo-diploid species can generally be made by the standard formula for sex-linked genes (see review by Caballero, 1994). Some situations occur, however, where reproduction of these species is more complex than assumed by the simplest models. For example, in many eusocial Hymenoptera species, males can be produced by workers rather than only by queens. Predictions of $N_{e}$ for this scenario have been developed by Nomura and Takahashi (2012).

\section{Populations under selection}

In the absence of selection or when selection acts on a non-inherited trait, the effective size is simply a function of the variance of the number of offspring per parent, as in Equation (5). However, predictions of $N_{e}$ are more complicated when selection acts on an inherited trait, such as when artificial selection is carried out for a quantitative trait in animal or plant breeding, or when natural selection acts on fitness traits against deleterious mutations or in favour of advantageous ones. In these scenarios, the drift process is amplified over generations because the random associations originated in a given generation between neutral and selected genes remain in descendants for a number of generations until they are eliminated by segregation and recombination. This problem was first addressed by Robertson (1961) and later on by other authors (for example, Wray and Thompson, 1990; Woolliams et al., 1993; Santiago and Caballero, 1995) for directional selection in quantitative traits. Extensions of the model were made later for populations under natural selection, linkage, overlapping generations and animal breeding schemes, as will be reviewed below.

Selection assuming unlinked genes. When selection acts on an inherited trait, changes in gene frequency at a focal neutral locus are positively correlated over generations because the selective values randomly associated with the neutral locus are not completely removed by segregation and recombination from one generation to the next. For unlinked genes and weak selection, the random association generated by sampling in a single generation is halved in consecutive generations by segregation and recombination. Therefore, the accumulative selective association has a limiting value $Q=$ $\sum_{i=0}^{\infty}(1 / 2)^{i}=2$ times the value of the original random association (Robertson, 1961), and the corresponding variance of the long-term contributions of copies of the neutral gene will increase by a factor $Q^{2}$. With regards to drift, the effective variance of contributions of individuals (with average 2) increases owing to selection by the same factor up to $4 Q^{2} C^{2}$, where the term $C^{2}$ is the genetic variance of the individual trait measures (for the quantitative trait subject to artificial selection or fitness-related traits in the case of natural selection) relative to the mean of the trait in the population. This variance has to be added to the expected variance of random contributions not caused by selection $\left(S_{k}^{2}\right)$ to predict the total variance of contributions. In reality, the associations are also reduced each generation to a proportion equal to the fraction of genetic variance remaining after 
selection $(G)$, which, in turn, can be increased by the correlation between the selective advantages of male and female parents $(r)$, and the series becomes

$$
Q=\sum_{i=0}^{\infty}[G(1+r) / 2]^{i}=\frac{2}{2-G(1+r)}
$$

(Santiago and Caballero, 1995). In the case of partial selfing (or partial full-sib mating), the term $r$ in Equation (12) should be replaced by $\beta$ (the proportion of inbred matings), because the correlation between the expected selective values of males and females $(r)$ is approximately 1 for inbred matings (which take place with proportion $\beta$ ) and approximately 0 for non-inbred matings, that is, $Q=2 /(2-G(1+\beta))$.

Therefore, the equation accounting for selection as an extension of Equation (5) is

$$
N_{e}=\frac{4 N}{2(1-\alpha)+\left(S_{k}^{2}+4 Q^{2} C^{2}\right)(1+\alpha)} .
$$

Nomura (1999b, 2005a) showed that Equation (13), obtained by a genetic drift approach, could also be derived from an inbreeding approach by considering the variance of long-term contributions as used by Wray and Thompson (1990) and Wray et al. (1990), when appropriate corrections are made in the latter (see also Woolliams and Bijma, 2000).

The application to different numbers of males and females was given by Santiago and Caballero (1995). That equation, however, lacked the same covariances as the equation without selection, as shown by Nomura (1997a) and Wang (1998). For random mating $(\alpha=0)$ and Poisson distribution of family sizes $\left(S_{k}^{2}=2\right)$, Equation (13) reduces to the simplest expression (Robertson, 1961),

$$
N_{e}=\frac{N}{1+Q^{2} C^{2}} \text {. }
$$

Equation (14) can be expressed in terms of heritability $\left(h^{2}\right)$ of fertility, as shown by Nei and Murata (1966) and Nomura (2002b). Let $V_{k}$ be the observed variance of family sizes, which would be $V_{k}=$ $S_{k}^{2}+4 C^{2}$ if the decay in the cumulative effect of selection is ignored (that is, $Q=2)$. The first term, $S_{k}^{2}=V_{k}\left(1-h^{2}\right)$, is the non-heritable component of this variance, and the second term, $4 C^{2}=V_{k} h^{2}$, is the heritable component. Thus substituting these into Equation (14) yields

$$
N_{e}=\frac{4 N}{2+\left(1+3 h^{2}\right) V_{k}}
$$

(Nei and Murata, 1966). The extension of Equation (15) to dioecious populations was developed by Nei and Murata (1966) assuming random union of gametes. A more general equation was developed by Nomura (2002b), who also suggested a form of the equation that avoids estimating the heritability,

$$
N_{e}=\frac{4 N}{2+V_{k}+3\left(\operatorname{cov}_{k, m}+\operatorname{cov}_{k, f}\right)},
$$

where $\operatorname{cov}_{k, f}$ and $\operatorname{cov}_{k, m}$ are the offspring-mother and offspring-father covariances of sibship size, respectively.

The prediction of effective population size under selection with overlapping generations was considered by Nomura (1996) and Bijma et al. (2000). As for the non-selection case (Hill, 1979), $N_{e}$ is the same as that for populations with discrete generations having the same nonselective and selective components of variance in lifetime progeny numbers and the same number of individuals entering the population each generation. Another interesting result is that the average age of parents in populations under selection is smaller than that in populations under no selection, as, in the former, younger parents tend to have higher selective advantages.

Genetic marker data can be used to assist selection (that is, markerassisted selection (MAS)) for a quantitative trait. Nomura (2000) investigated the predictive equation of $N_{e}$ in this scenario and showed that it depends on the relative values of the genetic $(r, 0.5$ for full-sib families) and phenotypic $(t)$ correlation between family members, where $t \approx h^{2} / 2+c^{2}, h^{2}$ is the trait heritability and $c^{2}$ is the fraction of the phenotypic variance owing to the shared common environments of family members. When an index $(I)$ is considered using individual phenotype $(P)$ and molecular marker $(M)$ information with given weights, that is, $I=B_{P} P+B_{M} M$, the effective size with MAS is reduced relative to that for phenotypic selection alone (Equation (14)) when $t<r$ and is increased when $t>r$.

The prediction of effective population size under index selection was addressed by Wray et al. (1994), Caballero et al. (1996b) and Nomura (1998b, 2005a). Assume truncation selection is carried out based on an index selection of the individual phenotype $(P)$ and the mean phenotype of its full-sib family $\left(P_{\mathrm{f}}\right.$, including the individual), $I=B_{w}\left(P-P_{f}\right)+B_{b}\left(P_{f}\right)$, where $B_{w}$ and $B_{b}$ are the corresponding selection weights. The effective size can then be predicted using Equation (14), where $Q=2 /\left(1+k B_{b}\right)$ and $C^{2}=S_{k}^{2}+4 i^{2}\left(\rho_{I}-\rho_{A}\right)+4 i^{2} \rho_{A}$, where $\rho_{I}$ is the correlation of full sibs for the index values, $\rho_{A}$ is the correlation of full sibs owing to the breeding value of the parents, $k=i(i-x), i$ is the selection intensity and $x$ is the truncation point in the standardised normal distribution. This predictive equation corrects a typographical error in a sign in the equation of Caballero et al. (1996b, p. 77). When the whole pedigree information is available, estimation of breeding values can be made by Best Linear Unbiased Prediction selection. Predictions of the effective size under this selection method were investigated by Nomura et al. (1999) Bijma and Woolliams (2000) and Bijma et al. (2001).

Other extensions for the prediction of the effective population size under selection refer to sex-linked loci (Nomura, 1997b; Wang, 1998), gynodioecious species (that is, species which have both hermaphrodite and female individuals, Laporte et al., 2000), open nucleus schemes (Nomura, 1997c; Bijma and Woolliams, 1999) and selection on traits affected by maternal effects (Rönnegård and Woolliams, 2003).

Selection at linked loci. The above formulations predict the rates of inbreeding that are usually calculated by tracing paths in genealogies of individuals. However, the real rates of inbreeding are expected to be larger than those predictions when selection acts on a system of linked genes. The reason for this is that the two gene copies at a neutral locus in an individual have different probabilities of propagation to the next generation, because they are embedded in homologous chromosomes with different alleles at linked selected loci. The problem of predicting $N_{e}$ in populations under purifying selection with linkage (the background selection model; Charlesworth, 2013) was addressed by Hudson and Kaplan (1995) and Nordborg et al. (1996) focussing on the effect of selection on nucleotide diversity, by Santiago and Caballero (1998) analysing the cumulative effect of selection over generations with a genetic drift approach (the Robertson effect) and by Nicolaisen and Desai $(2012,2013)$ using the coalescence theory. All these papers derived the same equation for the asymptotic $N_{e}$, which is a function of the haploid deleterious mutation rate $U$, the effect $s$ of mutations and the length $L$ of the whole-genome or genome segment given in Morgans,

$$
N_{e}=N e^{-U /(s+L / 2)} .
$$


This equation is the exponential version of Equation (14), $N_{e}=N e^{-Q^{2} C^{2}}$, which was derived from the multiplicative fitness model assumed under background selection. Here $C^{2}=U s$ is the variance for fitness and the cumulative term, $Q$, for a rate of recombination $c$ between the neutral and selected loci, is $Q_{c}=$ $\sum_{i=0}^{\infty}[(1-s)(1-c)]^{i} \approx 1 /(s+c)$ (Santiago and Caballero, 1998). If the focal neutral locus is located in the middle of the genome segment and the selected loci are uniformly scattered, the average value of the $Q_{c}^{2}$ terms over the segment is $Q^{2}=1 /(s(s+L / 2))$. Substituting this and $C^{2}=U s$ in Equation (14), we obtain Equation (17).

It is important to note that this equation predicts the magnitude of drift or inbreeding in the long term. For the focal neutral allele, this magnitude is effectively reached after a number of generations counted since it first appeared by mutation. Until that moment, drift at the neutral locus is expected to increase with time. The increasing drift acting on neutral mutations in consecutive generations can be predicted by the partial $N_{e(t)}$ values for generation $t$ forward in time that can be calculated using the partial cumulative terms $Q_{c(t)}=\sum_{i=0}^{t}[(1-s)(1-c)]^{i}=\left(1-e^{-(s+c) t}\right) /(s+c)$ (Santiago and Caballero, 1998). An equivalent conclusion was reached by Nicolaisen and Desai $(2012,2013)$ from the point of view of the coalescent process. The consecutive $N_{\mathrm{e}(-t)}$ values that predict the increasing probability of coalescence under selection $t$ generations backwards in time (thus the negative sign) reach an asymptotic value given by Equation (17) and the predictions of the partial $N_{e}$ values given by both methods, forward and backward, are exactly the same for any generation $t$,

$$
N_{e(t)}=N_{e(-t)}=N e^{-\frac{U s}{L / 2} \int_{0}^{L / 2} Q_{c(t)}^{2} d c} .
$$

Illustrations of the decline in $N_{e(t)}$ over generations are given in Figure 3 of Santiago and Caballero (1998) and Figure 3 of Nicolaisen and Desai (2013). This shows that the distortion of coalescent genealogies under selection and the cumulative effect of selection over generations are both specular images of the same process. Moreover, the pattern of neutral variation in populations under selection can be predicted by accumulating the expected distributions of neutral mutations originated in all the previous generations with the corresponding consecutive effective sizes given by the $N_{e(t)}$ values (Santiago and Caballero, 1998; Nicolaisen and Desai, 2012, 2013). This means that the intensity of genetic drift varies over mutations that occurred at different generations backward in time. Therefore, strictly speaking, there is not a single $N_{e}$ value representing the intensity of the drift process and, consequently, the amount and spectrum of variation under selection, even in populations at mutation-selection-drift equilibrium.

If mutations are advantageous (selective sweep model), predictions are generally cumbersome, because the genetic variance at selected loci is a function of the gene frequencies. Moreover, the stochastic distribution of selective sweeps over time is far away from the constant flux of variation usually assumed to simplify derivations. Wiehe and Stephan (1993) and Gillespie (2000) derived equations for the expected heterozygosity at neutral loci using a model in which recurrent favourable mutations pass quickly through the population to fixation, wiping out linked variation. The first key simplification in these derivations is that the time of fixation of favourable mutations is so short relative to the timescale of genetic drift that it can be considered as occurring instantaneously. The second simplification is that the rate of recovery of neutral variation after a selective sweep is very slow when compared with the rate of occurrence of the sweeps. The recurrent substitutions lead to a roughly constant level of neutral variation in a stochastic process that is often referred to as 'genetic draft'. A simple solution for $N_{e}$ can be obtained by directly applying the concept of variance of long-term contributions to an evolutionary timescale:

$$
N_{e}=\frac{N}{1+2 N \theta y^{2}}
$$

(Gillespie, 2000), where $\theta$ is the rate of selective sweeps and $y$ is the final frequency of the neutral copy that was initially associated with the favourable mutation when it first appeared. The frequency of this neutral copy is expected to increase to $N y$ copies after the sweep, and the frequency of each of the other $2 N-1$ copies is expected to be reduced to $(1-y)$ copies. Therefore, the variance of the expected longterm contributions for a single selective sweep is about $2 \mathrm{Ny^{2 }}$. As selective sweeps occur at a rate $\theta$, the second term of the denominator in Equation (19) is the total variance of the expected long-term contributions, that is, $Q^{2} C^{2}$ in Equation (14).

\section{Effective population size in conservation practices}

The concept of effective size is key to conservation genetic practices, as it summarises the past history of the population regarding inbreeding and genetic drift and provides the prospects for the sustainability of the population if the current effective size is maintained in the future. The effective population size is directly related to the statistics widely used to monitor conservation breeding schemes, such as the number of genome equivalents $N_{g e} \approx N_{e} / t$ (Lacy, 1995), where $t$ is the number of generations of management.

Minimising the loss of genetic variation is one of the main objectives of captive breeding programmes. This is achieved through minimising genetic drift and, therefore, maximising $N_{e}$. A classical strategy to follow is the equalisation of family sizes. By choosing one couple from each pair of parents, the variance of parental contributions is null $\left(S_{k}^{2}=0\right)$, and from Equation (1), $N_{e} \approx 2 N$ (Wright, 1938; Crow, 1954), which is twice the effective size of an unmanaged population with a Poisson distribution of family size. This is known as minimal inbreeding and it is the recommended procedure for applications in germplasm collection and regeneration in plants (see, for example, Vencovsky et al., 2012). However, effective sizes $>2 \mathrm{~N}$ can actually be obtained by population subdivision (Wright, 1943; Wang and Caballero, 1999) and other strategies, as shown below. The extension of the strategy in the case of different numbers of males and females was developed by Gowe et al. (1959). In their selection scheme, each male contributes one son and $r_{f m}=N_{f} / N_{m}$ daughters, and each female contributes one daughter and has a probability of $N_{m} / N_{f}$ of contributing one son. Thus $S_{f m}^{2}=\left(N_{m} / N_{f}\right)\left[1-\left(N_{m} / N_{f}\right)\right]$ and all of the other variances and covariances in Equation (2) are 0. Substituting these into Equations (2) and (3) gives

$$
N_{e}=\frac{16 N_{m} N_{f}}{3 N_{f}+N_{m}} \text {. }
$$

Compared with no selection (random Poisson distribution of the number of offspring per parent, Equation (4)), this scheme can increase $N_{e}$ by a proportion of $\left(r_{f m}+3\right) /\left(3 r_{f m}+1\right)$. When the female/ male ratio, $r_{f m}=N_{f} / N_{m}$, is 2 , for example, $N_{e}$ is increased by $71.4 \%$.

Wang $(1997 \mathrm{c})$ proposed an alternative design that produces further increases in $N_{e}$ of about $17 \%$ when $r_{f m}=2$. In this scheme, among the $r_{f m}$ females mated with each male, one is selected at random to contribute one son and each of the remaining $r_{f m}-1$ females contributes one daughter. In this scenario, $S_{f m}^{2}$ is as for Equation (20), but $S_{f f}^{2}=2 N_{m} / N_{f}$, and a negative covariance is induced between the numbers of male and female offspring from female parents, 
$S_{f m, f f}=-N_{m} / N_{f}$. Substituting these terms into Equations (2) and (3),

$$
N_{e}=\frac{16 N_{m} N_{f}^{2}}{3 N_{f}^{2}+2 N_{m}^{2}-N_{m} N_{f}} .
$$

The benefit of this scheme over that from Gowe et al. (1959) is decreased as $r_{f m}$ gets larger. For sex-linked loci, a benefit is also produced if males are the heterogametic sex. The above equations refer to random mating of parents. Wang (1997c) also proposed a system of non-random mating in which each male is mated with one of the groups of half-sib females who are not sisters of the male. This is a sort of population subdivision where the half-sibs are like 'subpopulations' and there is random migration of males and no migration of females among the 'subpopulations' (see Wang and Caballero, 1999). The mating scheme can further increase $N_{e}$ over that predicted by Equation (21).

Wang (1997c) method applies to a single generation. Sánchez et al. (2003) extended the method across generations to account for longterm contributions, further improving its efficiency. With the availability of pedigree or molecular marker information, a more general method, based on finding the contributions from parents to progeny that minimise the average coancestry among the progeny (minimum coancestry contributions), is the most widely proposed criterion to maintain genetic diversity (Toro and Pérez-Enciso, 1990; Ballou and Lacy, 1995; Fernández et al., 2003; Meuwissen, 2007). This method has been shown to minimise the variance of the long-term contributions from ancestors to descendants and, therefore, to maximise effective population size (Caballero and Toro, 2000, 2002).

The above methods are all designed to reduce the variation in family sizes, the term $S_{k}^{2}$ in Equation (1) and its corresponding components when the numbers of males and females are different (Equation (2)). It is also possible to increase the effective population size by decreasing the Mendelian segregation variance, which is represented by the constant term ' 2 ' in Equation (1). This latter can be achieved by the use of MAS to minimise the variation in contribution between the paternally and maternally derived genes at a locus (Wang and Hill, 2000). Thus, for example, for equal numbers of males and females and equalisation of individual contributions, $N_{e}$ can be expressed as

$$
N_{e}=\frac{2 N}{P_{m, m f}+P_{f, m f}},
$$

where $P_{m, m f}\left(P_{f, m f}\right)$ is the probability that the two genes coming from the male (female) parent and contributing to their male and female progeny are copies of the same gene. By MAS, it is possible to reduce these probabilities below the value of 0.5 expected under no control of Mendelian segregation, depending on the amount of marker information, the genome size and the number of marker-genotyped offspring per family, achieving values of $N_{e}>2 N$. MAS can also be used in a more general framework of different numbers of males and females to minimise global genetic drift and inbreeding (Wang, 2001a).

An alternative and complementary method is to use reproductive technologies for meiosis manipulation, such as in vitro culture of premeiotic germ cells and microinjection of primary spermatocytes into oocytes. By using more than one gamete from a single meiosis, variation from Mendelian segregation can be partially or completely removed (Santiago and Caballero, 2001). Thus, for example, if equalisation of family sizes is carried out and the gametes from both male and female parents are managed to come from the same meiosis in each case, the resulting effective size becomes $3 N$, rather than the typical $2 N$.
The control of the increase in inbreeding and genetic drift in conservation programmes is mainly addressed by reducing the variances of genetic contributions between paternally and maternally derived genes within and between individuals by equalising family sizes and minimising Mendelian segregation variance, as discussed above. A minor contribution to this control can also be achieved by the avoidance of inbred matings and other types of non-random mating. The simple avoidance of sib mating has a very minor effect (Wang, 1997d) and methods such as the maximum avoidance of inbreeding proposed by Wright (1921) have a higher, although still relatively low, impact. These can be carried out after the design of parental contributions has been implemented (Caballero et al., 1996a; Meuwissen, 2007). Alternatively, avoidance of inbreeding and optimal parental contributions can be realised in a single step (Fernández et al., 2004) by the so-called mate selection method. Systems of mating involving circular (half-sib mating) (Kimura and Crow, 1963b; Theodorou and Couvet, 2010) or rotational schemes (Nomura and Yonezawa, 1996) generally reduce the ultimate rate of inbreeding but at the cost of higher initial rates (Robertson, 1964), so that their use in conservation is not recommended because of the high risk of extinction from inbreeding depression. Some methods are of particular application in selection programmes, such as the compensatory mating proposed by Santiago and Caballero (1995), where individuals from large families are mated to individuals from small ones. This produces negative correlations between the drift caused by selection and the drift caused by sampling, partly counteracting the cumulative effect of selection represented by the term $Q$ in Equation (12). This system of mating in combination with maximum avoidance of inbreeding allows for a substantial reduction of inbreeding (Caballero et al., 1996a).

A conservation strategy of high relevance in fisheries is supportive breeding (Hare et al., 2011), where a population is typically divided into a captive and a wild group and the offspring of the captive group are released into the wild habitat to mix with the offspring from the wild group. Because the captive group (permanent or transitional) is bred to produce a lot of offspring that are released into the wild group at each generation, the variance in family size is greatly elevated artificially and thus the $N_{e}$ of the entire population is reduced. Ironically, the more successful the supportive programme is in augmenting the wild population, the greater the reduction in $N_{e}$ and the greater the loss of genetic diversity in the total population (supportive+wild). This paradox is overcome only when successful supportive breeding in augmenting the wild population is carried out over a long period of time such that the excessive drift and inbreeding in the initial generations of supportive breeding is compensated for by weaker drift and inbreeding in later generations because of the increase in census size. Ryman and Laikre (1991), Ryman et al. (1995) and Wang and Ryman (2001) have provided approximations for the inbreeding and variance effective sizes, respectively, which can be different in this case, with one generation of supportive breeding. Nomura (1998a) obtained an expression of $N_{e}$ from the change in coancestry, which agrees with the variance effective size, as expected. In this scenario, with various census sizes and a mixture of groups, predictions depend, however, on the generations considered (see Ryman et al., 1999).

For endangered species in the wild, estimating the effective population size and monitoring its changes over time is important in understanding the genetic health, evaluating the risk of inbreeding and inbreeding depression and thus the risk of extinction, assessing the effectiveness of the genetic managements (for example, human-aided migration/relocation, habitat protection or modification) and 
projecting the future demographic trajectories of the populations. Simulations (Tallmon et al., 2010) showed that monitoring the effective size is most often a more robust means of identifying stable and declining populations than monitoring census size. If a population is detected to have a small or declining $N_{e}$, the managers of the populations should be alerted to investigate the causes and to take effective measures for reversing the course. Using noninvasive sampling (for example, from faeces, feathers, hair and so on), genetic marker data can be obtained from a population even without observing (disturbing) the animals. The data can then be analysed normally, except for accounting for the possibility of genotyping errors and allowing for a high frequency of missing data (for example, Wang, 2004), in estimating $N_{e}$. For example, in a long-term monitoring project, Kamath et al. (2015) sampled and genotyped (at 20 microsatellite loci) 729 Yellowstone grizzly bears (Ursus arctos) born in the period 1962-2010 from an isolated and well-studied population in the Greater Yellowstone Ecosystem. They used the data to study the population demographic trajectories, estimating the changes in $N_{e}$ and generation interval, over this time period.

\section{METHODS FOR ESTIMATING THE EFFECTIVE POPULATION SIZE FROM GENETIC DATA}

Given the concepts of effective size, different approaches can be used to predict the effective size of a population from its demographic parameters, such as census size and variance of reproductive success. In parallel, different methodologies can also be developed to estimate the realized effective size of a population from its genetic properties revealed by genetic markers, such as temporal changes in allele frequency and linkage disequilibrium (LD).

Quite a few methods (Schwartz et al., 1999; Beaumont, 2003a; Wang, 2005; Palstra and Ruzzante, 2008; Luikart et al., 2010; Gilbert and Whitlock, 2015) have been developed and applied to estimating $N_{e}$ in widely different spatial and timescales, from ancient, past to current (parental) population sizes. Herein we focus on the effective size of the current generation or just a few generations in the past, as this timescale is the most relevant for conservation genetics (Luikart et al., 2010) and plant and animal breeding and is most likely to yield accurate estimates in current practices.

\section{Heterozygosity excess}

Compared with an infinitely large population at Hardy-Weinberg equilibrium, a population generated from a number of $N_{m}$ male parents and a number of $N_{f}$ female parents is expected to show a deficit of homozygotes and an excess of heterozygotes at a neutral locus when $N_{m}, N_{f}$ or both are small. This is because male and female parents are expected to have different allele frequencies owing to drift. The smaller the value of $N_{m}$ or $N_{f}$ the greater the difference between paternal and maternal allele frequencies and thus the greater the excess in heterozygosity of the offspring population. There is a simple functional relationship between the $N_{e}$ of the parental population and the amount of heterozygosity excess in the offspring population (for example, Robertson, 1965; Wang, 1996a). For a Wright-Fisher ideal population except for separate sexes with $N_{m}$ male and $N_{f}$ female parents, the heterozygosity excess is expected to be

$$
D \approx-\frac{1}{8 N_{m}}-\frac{1}{8 N_{f}}=-\frac{1}{2 N_{e}},
$$

where $N_{e}=4 N_{m} N_{f} f\left(N_{m}+N_{f}\right)$ is the effective size of the parental population given by Equation (4). The value of $D$ is negative, indicating an excess of heterozygosity and a corresponding deficit of homozygosity. For a non-ideal population with arbitrary distributions of family sizes, Equation (23) is still valid when $N_{m}$ and $N_{f}$ are replaced by $N_{e m}$ and $N_{e f}$, respectively, the effective numbers of male and female breeders.

Equation (23) suggests that measuring the heterozygosity excess, $D$, at a number of marker loci in a population yields an estimate of the parental population effective size. Pudovkin et al. (1996) proposed such a $N_{e}$ estimator by accounting for the sampling effect,

$$
\hat{N}_{e}=\frac{1}{2 \hat{D}}+\frac{1}{2(\hat{D}+1)},
$$

where the observed heterozygosity excess is estimated by $\hat{D}=\hat{H}_{e} /\left(\hat{H}_{e}-\hat{H}_{o}\right), \hat{H}_{e}=2 \hat{p}(1-\hat{p})$ is the expected heterozygosity from the observed gene frequency $\hat{p}$ and $\hat{H}_{\mathrm{o}}$ is the observed heterozygosity. $\hat{D}$ is calculated for each allele in a multiallelic locus and for each locus, and the average value is used in Equation (24) (Luikart and Cornuet, 1999). The accuracy of the estimator was evaluated by Pudovkin et al. (1996) using simulations and was applied to a few empirical data sets (Luikart and Cornuet, 1999). The method is simple and is implemented in several computer programs (for example, Zhdanova and Pudovkin, 2008; Jones and Wang, 2010; Do et al., 2014). However, the method has a low precision and accuracy, frequently providing infinitely large estimates of $N_{e}$ for small populations. The estimator is also highly sensitive to non-random mating (for example, population subdivision, close relative mating), which also causes deviation from Hardy-Weinberg equilibrium. Its poor performance renders it useless in applications to empirical data set analysis, except when the actual population size is very small and marker information is ample.

\section{Linkage disequilibrium}

In a large unselected random mating population, alleles are independent within and between loci, producing Hardy-Weinberg equilibrium and linkage equilibrium. In a finite population, however, random genetic drift leads to associations between alleles at a locus and between alleles of different loci. The former results in heterozygosity excess, and the latter leads to gametic LD. In addition to drift, LD can also be induced by factors such as migration and direct or indirect (for example, hitchhiking) selection (Hedrick et al., 1978). For neutral loci unlinked with selected loci in an isolated population under random mating, LD would come exclusively from genetic drift and can be used to estimate $N_{e}$ (Hill, 1981).

An LD estimator of $N_{e}$ for a random mating population at equilibrium is based on the formulation (Hill, 1981),

$$
E\left[r^{2}\right]=V[r]=\frac{(1-c)^{2}+c^{2}}{2 N_{e} c(2-c)}+\frac{1}{n}
$$

where $c$ is the recombination rate ( $c=1 / 2$ for unlinked loci), $r$ is the correlation of allele frequencies between two loci owing to LD and $n$ is the sample size (number of sampled individuals). In an equilibrium population, allele frequencies at two neutral loci are expected to be uncorrelated (that is, $E[r]=0$ ), such that the expectation of squared $r$, $E\left[r^{2}\right]$, is equal to the variance of $r, V[r]$. Equation (25) shows that $V[r]$ is composed of two distinctive parts. The first comes from genetic drift, determined by $N_{e}$ and linkage $c$. The second comes from sampling, determined by sample size $n$. Using the genotypes at a number of loci of $n$ sampled individuals, we can estimate $V[r]$, which can then be inserted in Equation (25) to obtain an estimate of $N_{e}$ if the recombination fraction $c$ between loci is known. Note that a slightly different expression for the population $V[r]$ (that is, the first part on the right-hand side of Equation 25) was derived by Sved (1971) from an identity by descent approach. For a dioecious population with 
monogamy, the right side of Equation (25) should be increased by $c /\left(2 N_{e} c(2-c)\right)$ (Weir and Hill, 1980).

Hill (1981) also derived the formula for the sampling variance of the estimator such that uncertainties of the $N_{e}$ estimates can also be evaluated. For the case of no linkage, Waples (2006) showed by simulations that the LD estimator can seriously underestimate $N_{e}$ when sample size is small. He derived empirical equations to correct for the bias caused by small sample sizes and showed by simulations that the accuracy of the modified estimator is comparable to the temporal method described in the next section. To facilitate the applications of the LD estimator, Waples and Do (2008) published a computer program, LDNE, and further evaluated its performance in comparison with the temporal method, using simulated data (Waples and Do, 2010). They concluded that, under similar conditions in terms of marker information and the actual population size, LD estimator can yield $N_{e}$ estimates that have equivalent or better qualities than the temporal estimators, except when the sampling interval of the temporal method is long.

The LD estimator is simple to calculate and requires just a single sample of multilocus genotypes instead of two or more samples, as is with the temporal method (see below). It is especially suitable for species with a long generation interval where obtaining two samples separated by a couple of generations means many years and for genetic monitoring (Schwartz et al., 2007) to track population trajectories on a yearly basis. As a result, the LD estimator has gained popularity in recent years (Palstra and Ruzzante, 2008; Luikart et al., 2010). However, some assumptions inherent to the LD estimators are often violated in real populations and as a result may lead to biased $N_{e}$ estimates. For example, it is assumed that LD is produced solely from the finite population size, and other confounding factors, such as nonrandom mating and population structure, are absent. Any departure from random mating (for example, an excess or deficit of close relatives mating including selfing) will affect LD and thus LD-based estimates of $N_{e}$. Waples et al. (2014) evaluated the effect of age structure on LD estimators and found that LD calculated from mixedage adult samples is overestimated and thus $N_{e}$ is underestimated in all of the 21 simulated species with different life tables. Similarly, the LD in a subpopulation is reduced by a constant and high rate of immigration and elevated by a low rate of immigration, compared with that of an isolated population of the same $N_{e}$. Therefore, as observed by Waples and England (2011) in their simulation study, LD calculated from a sample from a subpopulation leads to an overestimate or an underestimate of local $N_{e}$ when immigration rate is high or low, respectively. In the former case, the estimated local $N_{e}$ converges to the global $N_{e}$ of the entire population (Waples and England, 2011).

LD is highly dependent on the recombination rate between loci (Hill, 1981). Pairs of closer linked loci have higher LD and thus provide better information about $N_{e}$ (suitably defined in timescale) if the linkage information among loci is known. Although Hill (1981) derived his LD estimator of $N_{e}$ allowing for an arbitrary level of linkage and he advocated the use of linked markers, most often unlinked markers are used in practice because either truly unlinked markers are used or potentially linked markers are used but their linkage relationship is unknown. LD of markers of different recombination rates sheds light on the effective size of the population in different time periods in the past (Wang, 2005). Quite a few methods (Hayes et al., 2003; Barbato et al., 2015; Mezzavilla and Ghirotto, 2015; Saura et al., 2015) have been developed to exploit the LD information from many densely spaced markers on a chromosome segment in inferring the $N_{e}$ at different time points in the past.

\section{Temporal changes in allele frequency}

For an infinitely large population under Hardy-Weinberg equilibrium, both allele frequencies and genotype frequencies remain unchanged over time. In reality, these frequencies never stay constant and change systematically owing to the forces of mutation, selection and migration, stochastically due to the random force of genetic drift, or both. In the absence of the action of all of the systematic forces in a population, any observed allele frequency change must come solely from genetic drift and can thus be used to infer the rate of drift or the $N_{e}$ of the population. Based on this logic, Krimbas and Tsakas (1971) proposed to measure allele frequency changes at a number of marker loci between two temporally separated samples of individuals and thereby to estimate the $N_{e}$ of the population during the sampling interval. This so-called 'temporal method' was subsequently developed by many others in both (allele frequency) moment (for example, Nei and Tajima, 1981; Pollak, 1983; Waples, 1989) and likelihood (for example, Williamson and Slatkin, 1999; Anderson et al., 2000; Wang, 2001b; Berthier et al., 2002; Beaumont, 2003b; Laval et al., 2003) approaches.

Moment estimators calculate a standardised variance in the temporal changes of allele frequency, $F$, from marker genotypes in two temporally spaced samples. $F$ is essentially similar to Wright's $F_{S T}$, the differences being that $F$ measures the temporal differentiation for the same population and it also includes sampling effect. There are a few $F$ estimators (for example, Nei and Tajima, 1981; Pollak, 1983) available, the one being widely applied was derived by Nei and Tajima (1981). This estimator is calculated by

$$
\hat{F}=\frac{1}{k} \sum_{i=1}^{k} \frac{\left(x_{i}-y_{i}\right)^{2}}{\left(x_{i}+y_{i}\right) / 2-x_{i} y_{i}},
$$

for a locus with $k$ alleles, where $x_{i}$ and $y_{i}$ are the observed frequencies of allele $i$ in the first and second samples, respectively. For multiple loci, $\hat{F}$ is obtained by averaging single-locus estimates. The expectation of $\hat{F}$ depends on the sampling schemes (sampling with or without replacements) and is a function of $N_{e}$ and sample sizes to account for genetic drift and sampling effects. Solving the expectation equation of $\hat{F}$ for $N_{e}$ yields the temporal estimate of the (harmonic) mean $N_{e}$ during the sampling period (Nei and Tajima, 1981; Waples, 1989).

Moment estimators rely on the summary statistic, $F$, which is simple to calculate. However, they do not use the full allele frequency information and are thus less accurate than the probabilistic methods. The latter, likelihood or Bayesian, are much more complicated in statistical modelling and in computation. In general, temporal methods provide good estimates of $N_{e}$ when it is not large, sampling interval is not too short (for example, one generation) and the assumptions of the methods are satisfied, using a typical set of 10-20 microsatellites. Likelihood methods generally have higher accuracy and precision than moment methods, especially for markers with rare alleles, as verified by several extensive simulations (for example, Wang, 2001b; Berthier et al., 2002; Tallmon et al., 2004). They are, however, much more computationally demanding than moment methods, which complete an analysis almost instantly. Recently, the computational efficiency of likelihood methods has been improved substantially by Hui and Burt (2015), using a hidden Markov algorithm and applying continuous approximations to allele frequencies and transition probabilities. The new method can deal with $N_{e}$ values as high as several millions and is implemented in an R package called NB.

A constraint on the applications of temporal approaches is the requirement of at least two samples taken at one or preferably more generations apart. The longer this sampling interval, $t$, is, the stronger 
the drift signal will be in the temporal data and the more accurate the $N_{e}$ estimate will become. The extent of drift is proportional to $t$ and is inversely proportional to $N_{e}$. For the same population and the same sampling intensity (in terms of the number of markers, number of samples and number of individuals per sample), increasing the sampling interval $t$ could improve the accuracy of the temporal methods tremendously, as has been repeatedly verified by simulations (for example, Nei and Tajima, 1981). Moment estimators have especially low accuracy when $t$ is small (say, $t<3$ ) because of the weak drift signal and also because of the approximations made to the estimators. In practice, it is unfortunately difficult or unrealistic to increase $t$, especially for long-lived species having a long generation interval.

Compared with other $N_{e}$ estimating approaches, the temporal approach makes fewer assumptions and is more robust to some complications (realities) in real populations. For example, the approach is robust to population structure. It applies to a single unsubdivided population under non-random mating (including selfing) and to a subdivided population when sampling is representative and the aim is to estimate the $N_{e}$ of the entire subdivided population rather than that of a single subpopulation. It is also robust to age structure in a population with overlapping generations, so long as the sampling interval $t$ is large (for example, Nei and Tajima, 1981). When $t$ is small, however, Waples and Yokota (2007) showed by simulations that typical sampling regimes (sampling only newborns, only adults and all age classes in proportions) result in biased $N_{e}$ estimates. Jorde and Ryman (1995) developed a moment estimator of $N_{e}$ applicable to populations with overlapping generations. They derived an agestructure correction factor, which, when applied to the standard moment estimator for populations with discrete generations, leads to unbiased $N_{e}$ estimates for populations with overlapping generations. Unfortunately, however, the correction factor is a function of numerous age-specific survival rates and age-specific reproduction rates of the focal population. These rates are usually unknown. In fact, once all these rates are known for a population, the $N_{e}$ of the population can be calculated from standard $N_{e}$ prediction equations (for example, Felsenstein, 1971; Hill, 1972, 1979) without the need of genetic data. A method to calculate the $N_{e}$ of a population with overlapping generations from its demographic parameters has been implemented in an R-package Neff (Grimm et al., 2016).

The standard temporal approach for a single unsubdivided population was also extended to estimate the $N_{e}$ of a subpopulation that is connected to other subpopulations by gene flow (Wang and Whitlock, 2003). Although both drift and immigration change allele frequencies of a subpopulation, the detailed patterns of the changes are different between drift and immigration. Using temporal samples from a focal subpopulation and a sample from a large source population (the island-mainland model) or from two focal subpopulations (the island-island model), a moment estimator and a likelihood estimator can yield joint estimates of $N_{e}$ and migration rates, $m$. Simulations showed (Wang and Whitlock, 2003) that both moment and likelihood estimators gave reasonably good estimates of $N_{e}$ and $m$ under typical sampling intensities. However, no estimators are available for the more general case of multiple $(n>2)$ subpopulations. Part of the difficulty is with the number, $n^{2}$, of parameters to be jointly estimated, including $n$ effective sizes and $n(n-1)$ migration rates. More work is badly needed in this direction as spatial and temporal genotype data are becoming easy to collect thanks to the rapid developments in molecular technologies.

One of the assumptions in the temporal approach is the absence of selection so that any change in allele frequency comes solely from drift and thus indicates the effective size of the population. For most marker loci, the assumption is valid, especially for a small population over a short sampling interval of just a few generations. However, over a long period, some loci could be affected by adaptive selection or purging selection and their allele frequencies could change faster or slower than those of neutral loci unaffected by selection. Allele frequencies at neutral loci could also evolve faster or slower because of LD with those under selection. The temporal methods have been extended to estimate $N_{e}$ of a population and the selection coefficient, $s$, of a locus from time series data of allele frequencies (for example, Bollback et al., 2008; Mathieson and McVean, 2013; Foll et al., 2015). These methods are usually Bayesian, based on hidden Markov models to explain the observed allele frequency changes owing to drift and selection. How well these methods perform has yet to be checked, perhaps by a simulation study.

\section{Relatedness and relationship}

The pattern of genetic relatedness or relationship between individuals in a population has a direct functional relationship with the inbreeding effective size of the population (Wang, 2009). Two individuals taken at random from a population with a smaller $N_{e}$ will have a higher probability of sharing the same father, mother or both. More generally, the mean and variance in pairwise relatedness within a generation are expected to increase with decreasing $N_{e}$. Based on this logic, Nomura (2008) proposed a method to use the increase in average coancestry between two consecutive generations to estimate $N_{e}$. He showed by simulations that his coancestry method is more biased but more precise than the heterozygosity excess method. The overall accuracy (measured by mean squared errors) of the two methods is similar. A major problem which causes the bias of the method, as recognised by Nomura (2008), is that some non-sib pairs must be selected from a sample of individuals to act as reference in estimating the mean coancestry. The selection of non-sib pairs is difficult and somewhat subjective, because it is now well known that classifying dyads into even well-separated relationship categories, for example, full sibs, half sibs, parent offspring and unrelated, from pairwise relatedness estimates is highly error prone (for example, Blouin et al., 1996). Although many marker-based pairwise relatedness estimators are unbiased, they have high sampling errors with no exceptions (Wang, 2014).

A more robust and powerful method is to estimate the frequencies of half-sib (sharing a single parent) and full-sib (sharing both parents) dyads, $Q_{H S}$ and $Q_{F S}$, in a sample taken at random from a single cohort of a population (Wang, 2009; Waples and Waples, 2011). Wang (2009) derived a formula of $N_{e}$ in terms of half- and full-sib frequencies using both an inbreeding and a drift approach,

$$
\frac{1}{N_{e}}=\frac{1+3 \alpha}{4}\left(Q_{H S}+2 Q_{F S}\right)-\frac{\alpha}{2}\left(\frac{1}{N_{m}}+\frac{1}{N_{f}}\right) .
$$

The equation has the parameter $\alpha$ as in Equation (5), so that the $N_{e}$ for a population under non-random mating (for example, partial selfing) can be estimated. While $Q_{H S}$ and $Q_{F S}$ can be estimated from a sibship assignment analysis of the multilocus genotypes (Wang and Santure, 2009; Jones and Wang, 2010), $\alpha$ can be estimated from the same data with an $F_{S T}$-like approach (Wang, 2009). Alternatively, $\alpha$ can be assumed to be 0 for an outbred population when marker genotype frequencies do not deviate significantly from those expected under Hardy-Weinberg equilibrium. The difficulty comes from the estimation of the numbers of breeding males, $N_{m}$, and females, $N_{f}$, because sibship analysis generally makes no distinctions between paternal and maternal sibships from autosomal marker data, 
except in some specific situations (Wang, 2009). However, as detailed in Wang (2009), the bias brought about by the last term of Equation (27), $\frac{\alpha}{2}\left(\frac{1}{N_{m}}+\frac{1}{N_{f}}\right)$, is usually negligible because $\alpha$ is usually small and the estimate of $\frac{1}{N_{m}}+\frac{1}{N_{f}}$ by a sibship analysis is also not too far from its true value.

There are several advantages of this sibship approach compared with other single-sample approaches to $N_{e}$ estimation. First, sibship can be inferred more accurately than other quantities such as relatedness, which leads to more accurate estimates of $N_{e}$. Second, the approach applies to non-random mating populations, as an inbreeding coefficient $\alpha$ (Equation (5); equivalent to Wright's $F_{I S}$ ) can be calculated from the genotype data and incorporated into the $N_{e}$ estimate. Similarly, the approach is also robust to population subdivision, as discussed by Wang (2009). Third, it applies to diploid species, haplodiploid species, dioecious as well as monoecious species with selfing. Fourth, a great advantage is that it provides not only an estimate of the summary parameter $N_{e}$ but also some information about the numbers of male and female parents and variance in family sizes through the sibship assignment analysis. This detailed information is especially valuable for conservation management, as a low $N_{e}$ owing to high variance in family size or a low number of parents would imply different management strategies. Simulations verified that the approach is much more accurate than the heterozygosity excess method and is similar in accuracy to the temporal methods (Wang, 2009). However, it is unclear how its accuracy compares with that of the LD method. More work is needed to clarify this issue.

The above sibship frequency approach assumes a population with discrete generations. For a population with overlapping generations, the estimate provided by the sibship frequencies in a sample of singlecohort individuals is the effective number of breeders, $N_{b}$. This parameter summarises the effects of variation in reproductive success between age classes, between sexes and between individuals within an age-sex class on genetic drift in a single breeding season, instead of in a lifetime. It is less useful than $N_{e}$, and no population genetic equations are in terms of $N_{b}$. However, in the absence of an estimate of $N_{e}, N_{b}$ provides some information about the risks of inbreeding and loss of genetic variation in conservation populations (for example, Waples and Antao, 2014; Whiteley et al., 2015). For the case of overlapping generations, Wang et al. (2010) proposed a parentage assignment method to estimate the $N_{e}$ and generation interval from the sex, age and multilocus genotype information of a single sample of individuals taken at random from a population. Essentially, the method estimates the life table by parentage assignments, and both $N_{e}$ and generation interval are then calculated from the life table. Simulations showed that the method yields unbiased and reasonably accurate estimates of $N_{e}$ under realistic sampling and genotyping effort. Application of the method to empirical data yields sensible $N_{e}$ estimates that are supported by other sources of information from the population (Kamath et al., 2015).

\section{Multiple sources of drift/inbreeding information}

The above approaches to $N_{e}$ estimation use a single source of information, such as heterozygote excess, LD, temporal allele frequency changes and sibship/parentage frequencies. Each piece of information reflects a facet of the stochastic process (genetic drift or inbreeding) and combining multiple pieces of information may potentially allow for a better delineation of the process and thus yield a more accurate estimate of $N_{e}$. Tallmon et al. (2008) proposed to use approximate Bayesian computation $(\mathrm{ABC})$ to estimate $N_{e}$ from a sample of microsatellite genotypes. Their method, implemented in a computer program ONESAMP, calculates and uses eight summary statistics that are known to have functional relationships with $N_{e}$ from population genetic theory or simulations. These statistics include, among others, the number of alleles per locus, expected heterozygosity, LD, Wright's $F_{I S}$ and the mean and variance of multilocus homozygosity. In essence, the $\mathrm{ABC}$ approach simulates populations of different $N_{e}$ and tries to find the $N_{e}$ value that yields the same or similar summary statistics to those calculated from the real data. Tallmon et al. (2008) demonstrated this ABC approach by analysing an introduced increasing population of ibex Capra ibex.

It is arguable that the $\mathrm{ABC}$ approach uses more information than other approaches. On the one hand, it uses multiple sources of information such as heterozygosity, number of alleles and LD. However, on the other hand, for each source of information, it uses a summary statistic rather than the full information that is used by the probability methods (likelihood or Bayesian). Furthermore, it is unclear how these different summary statistics should be optimally weighted, given that these statistics are, apparently, highly correlated and may reflect the inbreeding and drift processes of different timescales. For a population changing in size, these different summary statistics are relevant for $N_{e}$ in different timescales. For example, $F_{I S}$ (similar to heterozygosity excess) is pertinent to the parental $N_{e}$, and LD implicates $N_{e}$ in the past few or more generations (depending on the linkage of the markers), while the number of alleles can be determined by the ancient $N_{e}$ many generations (in the order of $N_{e}$ or $1 / u$, whichever is smaller, where $u$ is mutation rate) ago. So far, an extensive simulation study to compare the accuracy of ABC and other approaches is lacking but is urgently needed.

\section{DISCUSSION}

Since the seminal work of Wright $(1931,1933)$, great progress has been made on the pivotal population genetic parameter, $N_{e}$, in its concepts (for example, inbreeding, variance, eigenvalue effective sizes and so on), its predictions for various species under different mating systems and population structures and its estimation methodologies using various marker information. In parallel, estimates of $N_{e}$, from both demographic and genetic data, have been made for many populations in the past 30 years, thanks to the rapid developments in both molecular technologies and statistical and computational methodologies.

Much work has been carried out to predict the effect of selection on $N_{e}$. However, developing useful predictive models on the effect of selection acting on an inherited trait remains difficult. The reason is that the impact of linked genes propagates over a number of generations, resulting in a long-term effect that is difficult to combine in a simple equation with parameters referred to only one generation time. Coalescence theory runs into similar difficulties in predicting $N_{e}$, because the probabilities of coalescence for consecutive generations are not independent under selection on an inherited trait. In addition to the variation of $N_{e}$ across generations, there is also variation over the genome. It is nowadays quite clear that there is a significant heterogeneity in levels and patterns of genetic variation across the genome caused by selection (Charlesworth, 2009; Gossmann et al., 2011), which complicates the inferences of $N_{e}$.

Another important remaining problem about selection is the interrelationship between $N_{e}$ and genetic variation. Most equations of $N_{e}$ are linear functions of the census size $N$ where genetic variation of the selected trait is an independent variable. However, genetic variation itself depends on $N_{e}$. Ignoring this fact is irrelevant for some purposes but is troublesome in some models of closely linked loci. This reciprocal dependence is on the basis of the Hill-Robertson effect (Hill and Robertson, 1966) and Mullerś ratchet (Haigh, 1978), both 
being different aspects of the same issue, an additional reduction of genetic variance owing to genetic drift induced by selection.

Demographic estimation of $N_{e}$ can be made by application of the predictive equations reviewed here when information on census sizes, variances of progeny numbers, type of mating system and other demographic data are available. The lack of these data and the increasing availability of genetic markers make the estimation of $N_{e}$ through genetic data to be, however, the leading procedure. Most factors affecting the populations in real situations imply a reduction of the effective size relative to the census size. In fact, the observed ratio $N_{e} / N$ has been found to be about 10-20\% (Frankham, 1995; Palstra and Fraser, 2012) on average in meta-analyses across many species and populations. Overall, these figures are in agreement with theoretical expectations obtained from some of the predictive equations presented in this review when fluctuations in population size are considered (Vucetich et al., 1997). However, this average $N_{e} / N$ ratio may be an overestimate, as marine species are under-represented in these metaanalyses and can have extremely low $N_{e} / N$ ratios.

Each $N_{e}$ estimation method with genetic data is based on a certain population genetic model and has a number of assumptions. It is important to realise that, when these assumptions are violated, which is unfortunately the rule rather than the exception in the real world, an estimation method may yield invalid or biased estimates of $N_{e}$. For example, most methods reviewed herein assume an isolated random mating population with discrete generations. Yet, in practice such populations are rare. The robustness of different methods has not been fully investigated.

Another important issue is the interpretation of the estimates obtained from a certain method. First, what is the spatial scale relevant to an $N_{e}$ estimate? Is it the effective size of the local population from which samples are taken or that of the metapopulation of which the sampled local population is a part? Apparently, the local and metapopulation effective sizes are very different in quantities and in applications. Local and metapopulation $N_{e}$ s signify the intensities of inbreeding and genetic drift processes at the local and global levels, respectively. A small local $N_{e}$ but a large global $N_{e}$ (that is, many small interconnected populations) and a large local $N_{e}$ but a small global $N_{e}$ (that is, a few large interconnected populations) have different ecological, evolutionary and conservation genetic implications.

Second, what is the temporal scale relevant to a $N_{e}$ estimate (Waples, 2005)? Is it the $N_{e}$ of the sampled population, of the parental population, of an ancestral population or the average $N_{e}$ over several/ many previous generations? The heterozygosity excess method and sibship methods estimate the parental population $N_{e}$, and temporal methods estimate the (harmonic) average $N_{e}$ over the generations in the sampling interval, while $\mathrm{LD}$ and $\mathrm{ABC}$ methods estimate the average $N_{e}$ over an unspecified number of previous generations (Wang, 2005). Of course, the temporal scale becomes irrelevant for a population with a constant unchanging demography. In practice, however, a natural population never stays the same.

Third, does the estimate refer to inbreeding or variance effective size? The question is irrelevant for the case of an isolated population with a constant demography or an incompletely subdivided (that is, with migration) population of constant size, as the inbreeding $\left(N_{e I}\right)$ and variance $\left(N_{e V}\right)$ effective sizes are the same. Otherwise, however, $N_{e I}$ and $N_{e V}$ can be dramatically different. A decreasing (increasing) population will always have an $N_{e I}$ greater (smaller) than $N_{e V}$, because the former and latter depend on the stochastic processes in the parental and the offspring generations, respectively (Crow and Kimura, 1970; Caballero, 1994; Wang and Caballero, 1999). Although usually unspecified in the original work describing the estimators, they estimate $N_{e I}, N_{e V}$ or a combination of both. The heterozygosity excess and sibship method estimate $N_{e I}$, while the temporal methods estimate $N_{e V}$. It is unclear what the LD and $\mathrm{ABC}$ methods estimate, but they likely estimate a combination of both $N_{e I}$ and $N_{e V}$.

Fourth, are the estimators unbiased and accurate when their underlying assumptions are met and are violated? Most estimators are not fully evaluated for their performances and statistical behaviours by simulation studies, especially those that are computationally intensive. In measuring the overall accuracy of an estimator, both precision and bias should be considered and better incorporated into a single measurement, such as mean squared errors. It is better to measure the mean squared errors of $1 /\left(2 N_{e}\right)$ rather than $N_{e}$, because the latter can be infinitely large and, more importantly, it is invariably $1 /\left(2 N_{e}\right)$ rather than $N_{e}$ that enters a population genetic equation (Wang and Whitlock, 2003). The dominating factor in determining accuracy is precision and bias when marker information is scarce and ample, respectively.

More work is also needed in developing estimators that make fewer restrictive assumptions and thus are more widely applicable to real populations. A common challenge to the current estimators is population genetic structure, including age structure (that is, overlapping generations) and spatial structure (that is, population subdivision with migration). Blindly applying an estimator developed for a single isolated population with discrete generations under random mating to an age- or space-structured population may yield unpredictable results.

Most current estimators have good performance for a population with a small $N_{e}$, partly because the drift or inbreeding signal is strong. For a large population with $N_{e}$ in the thousands or more, drift and inbreeding in the population is weak and is thus difficult to pick up by the typical sampling intensity (say, $\sim 100$ individuals, 10-20 microsatellites). With the rapid developments of genotyping (sequencing) technology, an increasing number of studies use many genome-wide markers to investigate population structure and demography. Use of thousands of single-nucleotide polymorphisms may compensate for the weak signal of stochasticity in a large population and thus may allow for a good estimate of large $N_{e}$. Future work is needed in evaluating current methods to estimate large $N_{e}$ using many markers. It can be reasonably assumed that, for an accurate estimate of $N_{e}$ (say, a narrow $95 \%$ confidence interval of roughly $\left(0.5 N_{e}, 2 N_{e}\right)$ ) by any efficient method, a sample size of individuals, of the total number of effective alleles across loci or of both in the order of $N_{e}$ might be required.

A related issue with large $N_{e}$ and many markers is the computational efficiency of likelihood or Bayesian estimators. Efficient algorithms, such as that proposed recently for the likelihood temporal estimator (Hui and Burt, 2015), are urgently needed to deal with big data sets. Another option is to exploit the modern multicore and multi-CPU computers and to parallel computer program codes using techniques such as MPI (Message Passing Interface) and OpenMP.

In some applications, the parameter, $N_{e}$, is all that is required in describing the current and predicting the future genetic variation in a population, and the demographic details that determine $N_{e}$ are irrelevant. In other applications such as the conservation management of endangered species, however, both $N_{e}$ and the demographic details of the population are useful in designing the most effective management to maintain the genetic diversity (Wang, 2009). When a population is estimated to have a small $N_{e}$ and thus is prone to the loss of genetic variation, we may ask what the causes are. Is the small $N_{e}$ caused by a small number of breeders, by a large variance in reproductive success among breeders, by an unbalanced sex ratio and 
so on? Different causes imply different optimal management strategies. The utility of future $N_{e}$ estimators could be improved if they provided joint estimates of $N_{e}$ and important demographic quantities, such as variance of reproductive success.

\section{CONFLICT OF INTEREST}

The authors declare no conflict of interest.

\section{ACKNOWLEDGEMENTS}

We thank Xav Harrison, the editor and five anonymous referees for helpful comments on previous versions of this manuscript. AC was funded by Ministerio de Economía y Competitividad (CGL2012-39861-C02-01), Xunta de Galicia (GPC2013-011) and Fondos Feder: 'Unha maneira de facer Europa'.

Allendorf FW, Luikart GH, Aitken SN (2013). Conservation and the Genetics of Populations. John Wiley and Sons: Chichester, West Sussex, UK.

Anderson EC, Williamson EG, Thompson EA (2000). Monte Carlo evaluation of the likelihood for Ne from temporally spaced samples. Genetics 156: 2109-2118.

Barbato M, Orozco-terWengel P, Tapio M, Bruford MW (2015). SNeP: a tool to estimate trends in recent effective population size trajectories using genome-wide SNP data. Front Genet 6: 109.

Ballou JD, Lacy RC (1995). Identifying genetically important individuals for management of genetic diversity in pedigreed populations. In: Ballou JD, Gilpin M, Foose TJ (eds). Population Management for Survival and Recovery. Columbia University Press: New York, USA.

Balloux F, Lehmann L (2003). Random mating with a finite number of matings. Genetics 165: 2313-2315.

Balloux F, Lehmann L, De Meeus T (2003). The population genetics of clonal and partially clonal diploids. Genetics 164: 1635-1644.

Beaumont MA (2003a). Conservation genetics. In: Balding DJ, Bishop M, Cannings C (eds). Handbook of Statistical Genetics, 2nd edn. Wiley: London, UK, pp 751-766.

Beaumont MA (2003b). Estimation of population growth or decline in genetically monitored populations. Genetics 164: 1139-1160.

Berthier P, Beaumont MA, Cornuet JM, Luikart G (2002). Likelihood-based estimation of the effective population size using temporal changes in allele frequencies: a genealogical approach. Genetics 160: 741-751.

Bijma P, Van Arendonk JA, Woolliams JA (2000). A general procedure for predicting rates of inbreeding in populations undergoing mass selection. Genetics 154: 1865-1877.

Bijma P, Woolliams JA (1999). Prediction of genetic contributions and generation intervals in populations with overlapping generations under selection. Genetics 151: 1197-1210.

Bijma P, Woolliams JA (2000). Prediction of rates of inbreeding in populations selected on best linear unbiased prediction of breeding value. Genetics 156: 361-373.

Bijma P, Van Arendonk JAM, Woolliams JA (2001). Predicting rates of inbreeding for livestock improvement schemes. J Anim Sci 79: 840-853.

Blouin MS, Parsons M, Lacaille V, Lotz S (1996). Use of microsatellite loci to classify individuals by relatedness. Mol Ecol 5: 393-401.

Bollback JP, York TL, Nielsen R (2008). Estimation of 2 Nes from temporal allele frequency data. Genetics 179: 497-502.

Caballero A (1994). Developments in the prediction of effective population size. Heredity 73: 657-679.

Caballero A (1995). On the effective size of populations with separate sexes, with particular reference to sex-linked genes. Genetics 139: 1007-1011.

Caballero A, Hill WG (1992). Effective size of nonrandom mating populations. Genetics 130: 909-916.

Caballero A, Santiago E, Toro MA (1996a). Systems of mating to reduce inbreeding in selected populations. Anim Sci 62: 431-442.

Caballero A, Toro MA (2000). Interrelations between effective population size and other pedigree tools for the management of conserved populations. Genet Res 75: 331-343.

Caballero A, Toro MA (2002). Analysis of genetic diversity for the management of conserved subdivided populations. Conserv Genet 3: 289-299.

Caballero A, Wei M, Hill WG (1996b). Survival rates of mutant genes under artificial selection using individual and family information. J Genet 75: 63-80.

Charlesworth B (2001). The effect of life-history and mode of inheritance on neutral genetic variability. Genet Res 77: 153-166.

Charlesworth B (2009). Fundamental concepts in genetics: effective population size and patterns of molecular evolution and variation. Nat Rev Genet 10: 195-205.

Charlesworth B (2013). Background selection 20 years on: The Wilhelmine E. Key 2012 invitational lecture. J Hered 104: 161-171.

Charlesworth B, Charlesworth D (2010). Elements of Evolutionary Genetics. Roberts \& Co: Greenwood Village, Colorado, USA.

Crow JF (1954). Breeding structure of populations. II. Effective population number. In: Kempthome O, Bancroft TA, Gowan JW, Lush JL (eds). Statistics and Mathematics in Biology. lowa State COIL Press: Ames, lowa, USA, pp 543-556.
Crow JF, Morton NF (1955). Measurement of gene frequency drift in small populations. Evolution 9: 202-214.

Crow JF, Denniston C (1988). Inbreeding and variance effective population numbers. Evolution 42: 482-495.

Crow JF, Kimura M (1970). An Introduction to Population Genetics Theory. Harper \& Row: New York, USA.

Do C, Waples RS, Peel D, Macbeth GM, Tillett BJ, Ovenden JR (2014). NeEstimator v2: reimplementation of software for the estimation of contemporary effective population size $(\mathrm{Ne})$ from genetic data. Mol Ecol Res 14: 209-214.

Engen S, Lande R, Saether BE (2005). Effective size of a fluctuating age-structured population. Genetics 170: 941-954.

Evans BJ, Charlesworth B (2013). The effect of nonindependent mate pairing on the effective population size. Genetics 193: 545-556.

Ewens WI (1979). Mathematical Population Genetics. Springer: Berlin, Germany.

Falconer D, Mackay TFC (1996). Introduction to Quantitative Genetics. Longman: Harlow, UK.

Felsenstein J (1971). Inbreeding and variance effective numbers in populations with overlapping generations. Genetics 68: 581-597.

Fernández J, Toro MA, Caballero A (2003). Fixed contributions designs versus minimization of global coancestry to control inbreeding in small populations. Genetics 165: 885-894.

Fernández J, Toro MA, Caballero A (2004). Managing individuals' contributions to maximize the allelic diversity maintained in small, conserved populations. Conserv Biol 18: $1-10$.

Fisher RA (1965). The Theory of Inbreeding, 2nd edn. Academic Press: New York, USA.

Foll M, Shim H, Jensen JD (2015). WFABC: a Wright-Fisher ABC-based approach for inferring effective population sizes and selection coefficients from time-sampled data. Mol Ecol Res 15: 87-98.

Frankham RD (1995). Effective population size/adult population size ratios in wildlife: a review. Genet Res 66: 95-107.

Frankham R, Ballou JD, Briscoe DA (2010). Introduction to Conservation Genetics. Cambridge University Press: Cambridge, UK.

Ghai GL (1969). Structure of populations under mixed random and sib mating. Theor Appl Genet 39: 179-182.

Gilbert KJ, Whitlock MC (2015). Evaluating methods for estimating local effective population size with and without migration. Evolution 69: 2154-2166.

Gillespie JH (2000). Genetic drift in an infinite population: the pseudo hitchhiking model. Genetics 155: 909-919.

Gossmann TI, Woolfit M, Eyre-Walker A (2011). Quantifying the variation in the effective population size within a genome. Genetics 189: 1389-1402.

Gowe RS, Robertson A, Latter BDH (1959). Environment and poultry breeding problems. 5. The design of poultry control strains. Poul Sci 38: 462-471.

Grimm A, Gruber B, Hoehn M, Enders K, Henle K (2016). A model-derived short-term estimation method of effective size for small populations with overlapping generations. Methods Ecol Evol (in press).

Haigh J (1978). The accumulation of deleterious genes in a population-Muller's Ratchet. Theor Popul Biol 14: 251-267.

Haldane JBS (1924). A mathematical theory of natural and artificial selection. H. The influence of partial self-fertilisation, inbreeding, assortative mating, and selective fertilisation on the composition of Mendelian populations, and on natural selection. Proc Camb Phil Soc (Biol Sci) (later Biol Rev) 1: 158-163.

Haldane JBS (1939). The equilibrium between mutation and random extinction. Ann Eugen 9: 400-405.

Hare MP, Nunney L, Schwartz MK, Ruzzante DE, Burford M, Waples RS et al. (2011). Understanding and estimating effective population size for practical application in marine species management. Conserv Biol 25: 438-449.

Hayes BJ, Visscher PM, McPartlan HC, Goddard ME (2003). Novel multilocus measure of linkage disequilibrium to estimate past effective population size. Genome Res 13: 635-643.

Hedrick P, Jain S, Holden L (1978). Multilocus systems in evolution. Evol Biol 11. 101-184.

Hill WG (1972). Effective size of populations with overlapping generations. Theor Popul Biol 3: 278-289.

Hill WG (1979). A note on effective population size with overlapping generations. Genetics 92: 317-322.

Hill WG (1981). Estimation of effective population size from data on linkage disequilibrium. Genet Res 38: 209-216.

Hill WG, Robertson A (1966). The effects of linkage on limits to artificial selection. Genet Res 8: 269-294.

Hudson RR, Kaplan NL (1995). Deleterious background selection with recombination. Genetics 141: 1605-1617.

Hui TYJ, Burt A (2015). Estimating effective population size from temporally spaced samples with a novel, efficient maximum-likelihood algorithm. Genetics 200: 285-293.

Jones OR, Wang J (2010). COLONY: a program for parentage and sibship inference from multilocus genotype data. Mol Ecol Res 10: 551-555.

Jorde PE, Ryman N (1995). Temporal allele frequency change and estimation of effective size in populations with overlapping generations. Genetics 139: 1077-1090.

Kamath PL, Haroldson MA, Luikart G, Paetkau D, Whitman C, Manen FT (2015). Multiple estimates of effective population size for monitoring a long-lived vertebrate: an application to Yellowstone grizzly bears. Mol Ecol 24: 5507-5521.

Kimura M, Crow JF (1963a). The measurement of effective population number. Evolution 17: $279-288$ 
Kimura M, Crow JF (1963b). On the maximum avoidance of inbreeding. Genet Res 4: 399-415.

Krimbas CB, Tsakas S (1971). The genetics of Dacus oleae V. Changes of esterase polymorphism in a natural population following insecticide control: selection or drift? Evolution 25: 454-460.

Lacy R (1995). Clarification of genetic terms and their use in the management of captive populations. Zoo Biol 14: 565-578.

Laporte V, Charlesworth B (2002). Effective population size and population subdivision in demographically structured populations. Genetics 162: 501-519.

Laporte V, Cuguen J, Couvet D (2000). Effective population sizes for cytoplasmic and nuclear genes in a gynodioecious species: the role of the sex determination system. Genetics 154: 447-458.

Laval G, SanCristobal M, Chevalet C (2003). Maximum-likehhood and Markov chain Monte Carlo approaches to estimate inbreeding and effective size from allele frequency changes. Genetics 164: 1189-1204.

Li CC (1976). First Course in Population Genetics. Boxwood: Pacific Grove, CA, USA.

Luikart G, Cornuet JM (1999). Estimating the effective number of breeders from heterozygote excess in progeny. Genetics 151: 1211-1216.

Luikart G, Ryman N, Tallmon DA, Schwartz MK, Allendorf FW (2010). Estimation of census and effective population sizes: the increasing usefulness of DNA-based approaches. Conserv Genet 11: 355-373.

Mathieson I, McVean G (2013). Estimating selection coefficients in spatially structured populations from time series data of allele frequencies. Genetics 193: 973-984.

Mezzavilla M, Ghirotto S (2015). Neon: An R package to estimate human effective population size and divergence time from patterns of linkage disequilibrium between SNPs. J Comput Sci Syst Biol 8: 037-044.

Meuwissen THE (2007). Operation of conservation schemes. In: Oldenbroek K (ed). Utilisation and Conservation of Farm Animal Genetic Resources. Wageningen Academic. Publishers: Wageningen, The Netherlands, pp 167-193.

Nagylaki T (1992). Theoretical Population Genetics. Springer: Berlin, Germany.

Nei M, Murata M (1966). Effective population size when fertility is inherited. Genet Res 8 : 257-260.

Nei M, Tajima F (1981). Genetic drift and estimation of effective population size. Genetics 98: 625-640.

Nicolaisen LE, Desai MM (2012). Distortions in genealogies due to purifying selection. Mol Biol Evol 29: 3589-3600.

Nicolaisen LE, Desai MM (2013). Distortions in genealogies due to purifying selection and recombination. Genetics 195: 221-230.

Nomura T (1996). Effective size of selected populations with overlapping generations J Anim Breed Genet 113: 1-16.

Nomura T (1997a). On the effective size of nonrandom mating populations under selection. Jpn J Biometrics 18: 1-11.

Nomura T (1997b). Effective population size for a sex-linked locus in populations under selection. Math Biosci 142: 79-89.

Nomura T (1997c). Prediction of effective population size in open nucleus breeding systems. J Anim Breed Genet 114: 333-347.

Nomura T (1998a). Effective population size in supportive breeding. Conserv Biol 13 $670-672$.

Nomura T (1998b). Effective population size and inbreeding under selection. Proceedings of the MAFF International Workshop on Genetic Resources Animal Genetic Resources II, 4-5 November 1998; Tsukuba, Ibaraki, Japan. National Institute of Agrobiological Resources: Tsukuba, Japan, pp 5-24.

Nomura T (1999a). Inbreeding coefficient and effective size of finite populations with a specified proportion of sib-mating. Jpn J Biometrics 83: 485-489.

Nomura T (1999b). On the methods for predicting the effective size of populations under selection. Heredity 83: 485-489.

Nomura T (2000). Effective population size under marker assisted selection. Jap $J$ Biometrics 21: 1-12.

Nomura T (2002a). Effective size of populations with unequal sex ratio and variation in mating success. J Anim Breed Genet 118: 297-310.

Nomura T (2002b). Effective size of populations with heritable variation in fitness. Heredity 89: 413-416.

Nomura T (2005a). Developments in prediction theories of the effective size of populations under selection. Anim Sci J 76: 87-96.

Nomura T (2005b). Effective population size under random mating with a finite number of matings. Genetics 171: 1441-1442.

Nomura T (2008). Estimation of effective number of breeders from molecular coancestry of single cohort sample. Evol Appl 1: 462-474.

Nomura T, Mukai F, Yamamoto A (1999). Prediction of response and inbreeding under selection based on best linear unbiased prediction in closed broiler lines. Anim Sci J 70: 273-281.

Nomura T, Takahashi J (2012). Effective population size in eusocial Hymenoptera with worker-produced males. Heredity 109: 261-268.

Nomura T, Yonezawa K (1996). A comparison of four systems of group mating for avoiding inbreeding. Genet Sel Evol 28: 141-159.

Nordborg M, Krone SM (2002). Separation of time scales and convergence to the coalescent in structured populations. In: Slatkin M, Veuille M (eds). Modern Developments in Theoretical Population Genetics: The Legacy of Gustave Malécot. Oxford University Press, Oxford, UK, pp 194-232.

Nordborg M, Charlesworth B, Charlesworth D (1996). The effect of recombination on background selection. Genet Res 67: 159-174.

Nunney $L$ (1993). The influence of mating system and overlapping generations on effective population size. Evolution 47: 1329-1341.
Orive M (1993). Effective population size in organisms with complex life-histories. Theor Pop Biol 44: 316-340.

Palstra FP, Ruzzante DE (2008). Genetic estimates of contemporary effective population size: what can they tell us about the importance of genetic stochasticity for wild population persistence? Mol Ecol 17: 3428-3447.

Palstra FP, Fraser DJ (2012). Effective/census population size ratio estimation: a compendium and appraisal. Ecol Evol 2: 2357-2365.

Pudovkin Al, Zaykin DV, Hedgecock D (1996). On the potential for estimating the effective number of breeders from heterozygote-excess in progeny. Genetics 144: 383-387.

Pollak E (1983). A new method for estimating the effective population size from allele frequency changes. Genetics 104: 531-548.

Pollak E (1987). On the theory of partially inbreeding finite populations. I. Partial selling. Genetics 117: 353-360.

Pollak E (2002). Eigenvalue effective population numbers for populations that vary cyclically in size. Math Biosci 177-178: 11-24.

Robertson A (1961). Inbreeding in artificial selection programmes. Genet Res 2 189-194.

Robertson A (1964). The effect of nonrandom mating within inbred lines on the rate of inbreeding. Genet Res 5: 164-167.

Robertson A (1965). The interpretation of genotypic ratios in domestic animal populations. Anim Prod 7: 319-324.

Rönnegård L, Woolliams JA (2003). Predicted rates of inbreeding with additive maternal effects. Genet Res 82: 67-77.

Ryman N, Laikre L (1991). Effects of supportive breeding on the genetically effective population size. Conserv Biol 5: 325-329.

Ryman N, Jorde PE, Laikre L (1995). Supportive breeding and variance effective population size. Conserv Biol 9: 1619-1628.

Ryman N, Jorde PE, Laikre L (1999). Supportive breeding and inbreeding effective number: Reply to Nomura. Conserv Biol 13: 673-676.

Sánchez L, Bijma P, Woolliams JA (2003). Minimizing inbreeding by managing genetic contributions across generations. Genetics 164: 1589-1595.

Santiago E, Caballero A (1995). Effective size of populations under selection. Genetics 139: 1013-1030.

Santiago E, Caballero A (1998). Effective size and polymorphism of linked neutral loci in populations under directional selection. Genetics 149: 2105-2117.

Santiago E, Caballero A (2001). Application of reproductive technologies to the conservation of genetic resources. Conserv Biol 14: 1831-1836.

Saura M, Tenesa A, Woolliams JA, Fernández A, Villanueva B (2015). Evaluation of the linkage-disequilibrium method for the estimation of effective population size when generations overlap: an empirical case. BMC Genomics 16: 922.

Schwartz MK, Tallmon DA, Luikart G (1999). Using genetics to estimate the size of wild populations: many methods, much potential, uncertain utility. Anim Conserv 2 321-323.

Schwartz MK, Luikart G, Waples RS (2007). Genetic monitoring as a promising tool for conservation and management. TREE 22: 25-33.

Sved JA (1971). Linkage disequilibrium and homozygosity of chromosome segments in finite populations. Theor Pop Biol 2: 125-141.

Tallmon DA, Luikart G, Beaumont MA (2004). Comparative evaluation of a new effective population size estimator based on approximate Bayesian computation. Genetics 167 977-988.

Tallmon DA, Koyuk A, Luikart G, Beaumont MA (2008). COMPUTER PROGRAMS: onesamp: a program to estimate effective population size using approximate Bayesian computation. Mol Ecol Res 8: 299-301.

Tallmon DA, Gregovich D, Waples RS, Baker CS, Jackson J, Taylor B et al. (2010). When are genetic methods useful for estimating contemporary abundance and detecting population trends?. Mol Ecol Res 10: 684-692.

Theodorou K, Couvet D (2010). Genetic management of captive populations: the advantages of circular mating. Conserv Genet 11: 2289-2297.

Toro MA, Pérez-Enciso M (1990). Optimization of selection response under restricted inbreeding. Genet Sel Evol 22: 93-107.

Vencovsky R, Chaves LJ, Crossa J (2012). Variance population size for dioecious species Crop Sci 52: 79-90.

Vucetich JA, Waite TA, Nunney L (1997). Fluctuating population size and the ratio of effective size to census population size. Evolution 51: 2017-3021.

Wakeley J (2008). Coalescent Theory. An Introduction. Ben Roberts: Greenwood Village, Colorado, USA.

Wakeley J, Sargsyan O (2009). Extensions of the coalescent effective population size. Genetics 181: 341-345.

Wang J (1996a). Deviation from Hardy-Weinberg proportions in finite populations. Genet Res 68: 249-257.

Wang J (1996b). Inbreeding and variance effective sizes for non-random mating populations. Evolution 50: 1786-1794.

Wang J (1996c). Inbreeding coefficient and effective size for an X-linked locus in nonrandom mating populations. Heredity 76: 569-577.

Wang J (1997a). Effective size and F-statistics of subdivided populations. I. Monoecious species with partial selfing. Genetics 146: 1453-1463.

Wang J (1997b). Effective size and F-statistics of subdivided populations. II. Dioecious species. Genetics 146: 1465-1474.

Wang J (1997c). More efficient breeding systems for controlling inbreeding and effective size in animal populations. Heredity 79: 591-599.

Wang J (1997d). Effect of excluding sib matings on inbreeding coefficient and effective size of finite diploid populations. Biometrics 53: 1354-1365. 
Wang J (1998). Effective size of populations under selection: some extensions. Proc 6th World Cong Genet Appl Livest Prod 26: 13-16.

Wang J (2001a). Optimal marker-assisted selection to increase the effective size of small populations. Genetics 157: 867-874.

Wang J (2001b). A pseudo-likelihood method for estimating effective population size from temporally spaced samples. Genet Res 78: 243-257.

Wang J (2004). Sibship reconstruction from genetic data with typing errors. Genetics 166 : 1963-1979.

Wang J (2005). Estimation of effective population sizes from data on genetic markers. Phil Trans $R$ Soc B 360: 1395-1409.

Wang J (2009). A new method for estimating effective population sizes from a single sample of multilocus genotypes. Mol Ecol 18: 2148-2164.

Wang J (2014). Marker-based estimates of relatedness and inbreeding coefficients: an assessment of current methods. J Evol Biol 27: 518-530.

Wang J, Caballero A (1999). Developments in predicting the effective size of subdivided populations. Heredity 82: 212-226.

Wang J, Hill WG (2000). Marker assisted selection to increase effective population size by reducing Mendelian segregation variance. Genetics 154: 475-489.

Wang J, Ryman N (2001). Genetic effects of multiple generations of supportive breeding. Conserv Biol 15: 1619-1631.

Wang J, Santure AW (2009). Parentage and sibship inference from multilocus genotype data under polygamy. Genetics 181: 1579-1594.

Wang J, Whitlock MC (2003). Estimating effective population size and migration rates from genetic samples over space and time. Genetics 163: 429-446.

Wang J, Brekke P, Huchard E, Knapp LA, Cowlishaw G (2010). Estimation of parameters of inbreeding and genetic drift in populations with overlapping generations. Evolution 64: 1704-1718.

Wang Y, Pollak E (2002). The effective number of population that varies cyclically in size. II. Overlapping generations. Math Biosci 179: 161-181.

Waples RS (1989). A generalized approach for estimating effective population size from temporal changes in allele frequency. Genetics 121: 379-391.

Waples RS (2005). Genetic estimates of contemporary effective population size: to what time periods do the estimates apply? Mol Ecol 14: 3335-3352.

Waples RS (2006). A bias correction for estimates of effective population size based on linkage disequilibrium at unlinked gene loci. Conserv Genet 7: 167-184.

Waples RS, Do C (2008). LdNe: a program for estimating effective population size from data on linkage disequilibrium. Mol Ecol Res 8: 753-756.

Waples RS, Do C (2010). Linkage disequilibrium estimates of contemporary Ne using SNPS and highly polymorphic molecular markers: an evaluation of precision and bias. Evol App/ 3: 244-262.

Waples RS, England PR (2011). Estimating contemporary effective population size on the basis of linkage disequilibrium in the face of migration. Genetics 189: 633-644.

Waples RS, Antao T (2014). Intermittent breeding and constraints on litter size: consequences for effective population size per generation $\left(N_{e}\right)$ and per reproductive cycle $\left(N_{b}\right)$. Evolution 68: 1722-1734.

Waples RS, Waples RK (2011). Inbreeding effective population size and parentage analysis without parents. Mol Ecol Res 11: 162-171.
Waples RS, Yokota M (2007). Temporal estimates of effective population size in species with overlapping generations. Genetics 175: 219-233.

Waples RS, Antao T, Luikart G (2014). Effects of overlapping generations on linkage disequilibrium estimates of effective population size. Genetics 197: 769-780.

Weir BS, Hill WG (1980). Effect of mating structure on variation in linkage disequilibrium. Genetics 95: 477-488.

Whiteley AR, Coombs JA, Cembrola M, O'Donnell MJ, Hudy M, Nislow KH et al. (2015). Effective number of breeders provides a link between interannual variation in stream flow and individual reproductive contribution in a stream salmonid. Mol Ecol 24: 3585-3602.

Whitlock M, Barton N (1997). The effective size of a subdivided population. Genetics 146: 427-441.

Wiehe THE, Stephan W (1993). Analysis of a genetic hitch-hiking model, and its application to DNA polymorphism data from Drosophila melanogaster. Mol Biol Evol 10: 842-854.

Williamson EG, Slatkin M (1999). Using maximum likelihood to estimate population size from temporal changes in allele frequencies. Genetics 152: 755-761.

Woolliams JA, Bijma P (2000). Predicting rates of inbreeding in populations undergoing selection. Genetics 154: 1851-1864.

Woolliams JA, Thompson R (1994). A theory of genetic contributions. Proc 5th World Cong Genet Appl Livest Prod 19: 127-134.

Woolliams JA, Wray NR, Thompson R (1993). Prediction of long-term contributions and inbreeding in populations undergoing mass selection. Genet Res 62: 231-242.

Wray NR, Thompson R (1990). Predictions of rates of inbreeding in selected populations. Genet Res 55: 41-54.

Wray NR, Woolliams JA, Thompson R (1990). Methods for predicting rates of inbreeding in selected populations. Theor Appl Genet 80: 503-512.

Wray NR, Woolliams JA, Thompson R (1994). Predicting of rates of inbreeding in populations undergoing index selection. Theor Appl Genet 87: 878-892.

Wright S (1921). Systems of mating. Genetics 6: 111-178.

Wright S (1931). Evolution in Mendelian populations. Genetics 16: 97-159.

Wright S (1933). Inbreeding and homozygosis. Proc Natl Acad Sci USA 19: 411-420.

Wright $S$ (1938). Size of population and breeding structure in relation to evolution. Science 87: 430-431.

Wright S (1939). Statistical Genetics in Relation to Evolution. Exposés de Biométrie et de Statistique Biologique. Herman \& Cie: Paris, France.

Wright S (1943). Isolation by distance. Genetics 28: 114-138.

Wright S (1969). Evolution and the Genetics of Populations, vol. 2, The Theory of Gene Frequencies. University of Chicago Press: Chicago, IL, USA.

Yonezawa K (1997). Effective population size of plant species propagating with mixed sexual and asexual reproduction system. Genet Res 70: 251-258.

Yonezawa K, Kinoshita E, Watano Y, Zentoh H (2000). Formulation and estimation of the effective size of stage-structured populations in Fritillaria camtschatcensis, a perennial herb with a complex life history. Genetics 54: 2007-2013.

Yonezawa K, Ishii T, Nagamine T (2004). The effective size of mixed sexually and asexually reproducing populations. Genetics 166: 1529-1539.

Zhdanova OL, Pudovkin Al (2008). Nb_HetEx: a program to estimate the effective number of breeders. J Hered 99: 694-695. 